\title{
Infosec-Tree Model: An Applied, In-depth, and Structured Information Security Model for Computer and Network Systems
}

\author{
Ricardo Villalón-Fonseca \\ CITIC-ECCI \\ Universidad de Costa Rica \\ San José, Costa Rica
}

\author{
Braulio J. Solano-Rojas \\ CITIC-ECCI \\ Universidad de Costa Rica \\ San José, Costa Rica
}

\author{
Gabriela Marín-Raventós \\ CITIC-ECCI \\ Universidad de Costa Rica \\ San José, Costa Rica
}

\begin{abstract}
Information security is a main concern in many fields of computer and information technologies, from software development, or network systems, to new or emerging technologies such as mobile, cloud computing, or social computing. Existing security standards and models usually focus on "what" has to be done about security, but they do not propose "how" to deal with the inherent complexity of assuring modern software systems or network infrastructures. Application of current security standards usually produce large check lists describing security countermeasures, but they lack a structured, in-depth and consistent process to define the information security requirements at different granularity levels of the system. As a consequence, security deployments may miss important security controls. We propose the Infosec-tree Model, a novel methodology with a hierarchical approach to guide that comprehensive assurance process for a computer or network system. Real use cases are presented, by applying our methodology to assure a private cloud being developed at the Universidad de Costa Rica (UCR).
\end{abstract}

\section{Introduction}

Information security for computers and network systems has held a central position on the interest of theoretical and practical researchers for many years. Security vulnerabilities arise everywhere, even on new and emerging technologies. For example, cloud computing is a modern, rapidly evolving service-oriented paradigm [1], [2], that offers capabilities for on-demand by-subscription usage, self-service, ubiquitous access, multi-tenancy, resource pooling, automatic elasticity, measured services, resiliency and more [2], [3], [4]. But these new functions come at the cost of additional security challenges and issues [5]. Its related security problems and concerns are ranked on top priority positions [4], [5], [6]. Some security risks are still the same that we have seen for many years, such as traffic eavesdropping, malicious intermediaries, or denial of services; but others are new or related to emerging technologies, such as insufficient authorization, virtualization attacks, or overlapping trust boundaries [3], [4], [6].

Existing security models and standards focus on providing risk analysis and policy definition. Most of them aim to define "what" should be done about security for a software system or technological infrastructure. Furthermore, current assurance methodologies [7], [8], [9], [10], [11], [12] also focus on strategies for risk analysis and security policy definition, but they do not provide an organized way to implement strategies and security mechanisms [25], using a systematic process to guarantee an in-depth, consistent and structured implementation of security requirements. There are models supporting security for specific systems or platforms, such as cloud computing [11], [12], [21], but they do not propose "how" to deal with the complexity, or how to organize security countermeasures for a large number of hardware and software components. Moreover, they do not provide a guiding process to execute and assure the entire platform, at the different granularity levels.

Strategies for risk analysis and policy definition are not enough to assure information for large or complex systems, like a cloud data center or a modern web application. They are composed of many interconnected elements, and they have different complexity levels. Also, they use a variety of hardware and software technologies, and there are many different security requirements. In these scenarios, it may not be easy to define a coherent and consistent set of security mechanisms to implement the required security services for the system. For example, it may not be clear how to define a complete set of mechanisms to avoid the lack of proper authentication on a large web application, or how to avoid the lack of proper encryption for passwords or sensitive information moving between any pair of devices in a cloud.

We argue that there is a gap between existing security models, and the process required to define "how" security should be implemented, mostly when assuring large and complex systems, or technological infrastructures. Traditional solutions such as security check lists and guidelines tend to be very large and unstructured. They may miss some security controls for the information being managed in the system, 
enabling security vulnerabilities. Therefore, check lists do not provide an appropriate guidance to clearly visualize and implement a large set of security requirements. Furthermore, security documentation tend to have a similar format, namely large lists describing many elements and aspects related to security, that may be hard to maintain over time.

Instead, we propose the Infosec-tree Model, an improved version of a previous work presented in [24], with a hierarchical whole-parts approach, to implement strategies and security mechanisms for assuring information being managed by hardware or software components in a system, and at different granularity levels. Higher levels of the tree define security mechanisms for larger components, while lower levels define specific security controls for smaller components. The entire tree provides an appropriate structure to integrate the security strategies through an in-depth, consistent, and structured assurance process.

This paper describes the information structures comprising the model, as well as an applied assurance process to define and implement strategies and security mechanisms, supported by a pre-defined set of security policies. The main contributions of this work are the model representing the system components and their security requirements, and the methodology to implement the assurance process.

We created a model and applied the methodology to assure a non-commercial institutional private computer cloud at the UCR, for the academic and administrative communities, to provide Infrastructure as a Service (IaaS) and Platform as a Service (PaaS), and Software as a Service (SaaS) in the future.

The document is structured as follows. Section 2 presents the underlying model and the methodology steps. Section 3 describes our experience at the UCR with study cases using the methodology. Section 4 provides some related work and a comparison of our methodology with other existing approaches. Last section provides some concluding remarks.

\section{Infosec-tree Model and Assurance Methodology}

We describe the goals and assumptions supporting our approach. Then, we define the model components required to apply the methodology. The methodology steps are included in the last subsection.

\subsection{Goals and Assumptions}

The main goal of our methodology is to guarantee an in-depth, consistent and structured implementation of security for an information system in a computer system or technological infrastructure composed of hardware and/or software. To reach this goal, we propose to create a hierarchical information structure representing the components of the system and their security requirements, starting with larger components, and going down into the structure to define smaller elements. The structure is arranged in a whole-parts pattern that supports the security perspective. This arrangement allows splitting a large and complex component and its related security problems in smaller and simpler cases, enabling security analysis to install security countermeasures at different levels of the hierarchical structure, in a systematic and granular way.

A second goal is to provide an applied security design tool to specify information security requirements and countermeasures for an entire computer system or technological infrastructure.

A third goal is to provide integral security by satisfying security requirements at different points in time with respect to the occurrence of security events.

- Preventive security to attend requirements before issues arises.

- Monitoring tools to detect security events as they occur.

- Response and recovery procedures to attend or fix the system state after security breaks.

Additionally, there are a few assumptions about the methodology. It is a tool to apply security principles and to implement security mechanisms, describing "how security should be implemented" but assuming a previously defined set of security policies. On the other side, it is neither a risk assessment, nor a policy definition process. Moreover, it is not a tool to define "what has to be done" about security, and it is not a vulnerability or threat analysis tool, but the generated documentation can help to guide a diagnostic process.

\subsection{Components of the Model}

The model is composed of a hierarchical information structure designed that supports the assurance process. First, we describe the main elements namely Infosec-tree and infosec-nodes. After that, we detail simpler elements, such as security triads, information states, security services, and assurance steps.

2.2.1. Infosec-tree. An Infosec-tree is a hierarchical structure describing all the system components with information security requirements in a system or infrastructure. The Infosec-tree is the largest component of the model, and is constructed using a whole-parts pattern. Elements at higher levels of the tree represent larger components of the system, and elements at lower levels represent smaller parts composing the larger elements above them.

The root element of the structure always represents the entire system we want to assure, independently on the size of the system. For example, an entire web application, or a full data center can be root nodes. We can decompose the root node into smaller parts, namely child nodes, and recursively each of them into smaller nodes, 
representing a set of subcomponents of its parent node. The decision about what the child nodes represent is assigned to the security engineer, and it depends on the security requirements. In all cases a child node should represent a component or subset of components of the parent node. The sum of the children should represent the same set of components included in the corresponding parent node, while respecting a whole-parts pattern structure.

For example, consider a small "data center" infrastructure composed of an "enclosure device" containing "blade servers". Additionally, consider a "switching device" to connect the servers to an external "storage device", and to allow connectivity to an external network. Furthermore, the storage device provides two logical services, namely storage area network (SAN) to supply directly connected hard disk storage for servers, and network attached storage (NAS) for network storage such as NFS or SMB. The resulting Infosec-tree is shown in Fig. 1.

Note that Fig. 1 contains different types of nodes. Enclosure, blades, switch and storage represent physical devices that share information using physical connections. They are physical elements of data center architecture. On the other side, SAN and NAS services provide a logical composition for the storage device, as opposed to physical, but they still preserve the required whole-parts pattern of the tree. A different approach would be to define the storage as composed of a set of physical hard disks, and that could be a valid design approach in a different context, but for the purpose of assuring this system it is better to use the logical approach.

In the general case, the criteria for dividing a node into its components is a decision of the security engineer, and it depends on risks, vulnerabilities, security requirements, physical and logical components of the system, and available security countermeasures.

Components of an Infosec-tree can be hardware or software, physical or logical, real or virtual, or a mix, as long as the structure created describes a whole-parts pattern for all the levels of the system being assured. Following with the example on Fig. 1, a physical blade server installed with hypervisor operating system for virtualization can be described as composed of a set of virtual networks, each of them represented by the virtual switches inside the hypervisor. Then, each virtual network can be composed of the virtual machines connected to it. In this example the parent node "blade" is real, but the subcomponents are virtual elements.

The granularity level of the components, and consequently the depth of the tree, is another relevant aspect of an Infosec-tree. It depends on how specific the security requirements are. An Infosec-tree with only a few levels is going to describe general security mechanisms, implemented at the big components of the system. A tree with more levels is going to provide detailed security controls for specific components. Also, the depth of the tree can

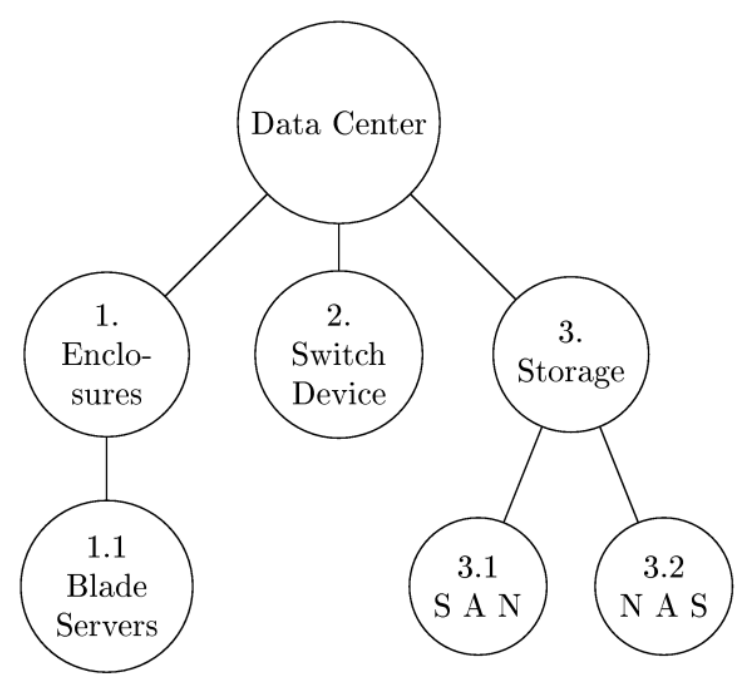

Figure 1. Infosec-tree example.

be different for each branch of the structure, enabling more specific levels of security as required.

After constructing the tree, we can establish security requirements and mechanisms for all or some nodes, including the root node if desired. Consider the root node described in Fig. 1, we can define confidentiality requirements for the entire data center by installing some security mechanism, such as a firewall, at the entry point of the infrastructure. That way, the entire system can protect information coming in and out of the data center. But we can extend the example, with additional requirements for children nodes, like implementing VLANs at the switching node, to isolate traffic for internal network segments, and to support confidentiality for information inside the data center.

We may gradually analyze more specific security requirements, going down into the tree, and adding countermeasures in a systematic and comprehensive way, while splitting each component into smaller parts, if required.

In summary, the key aspects to create the Infosec-tree structure are a) a whole-parts design to divide the system into components, b) a selection process to pick the right components to assure, according to the defined security requirements, and c) a granularity or detail level as a function of the security policies and depending on how strict they are applied during the assurance process.

2.2.2. Infosec-nodes. An infosec-node is an information structure to represent a node of the Infosec-tree. Infosec-nodes can represent hardware devices, real communication devices, virtual appliances, network services, and more. They can also be abstract components, such as an entire software application, a programming object, a set of software modules, or any other piece of software with capabilities to handle information.

As already mentioned, an infosec-node can be constructed with mixed types of components. For example, it can be a physical computer containing a set of virtual appliances, or it can be a communication device having virtual contexts. 
An infosec-node also describes the security requirements and countermeasures for information inside the node, or for information moving in and out of the node. Examples of security requirements are confidentiality required for a data center, integrity for stored information and transferred web pages of a web server, confidentiality and integrity for information stored in a hard disk, validation of parameters for a programming function, and so on.

Formally, an infosec-node contains the following elements:

- A node ID: a numeric value to identify the node. The node ID is used to refer to the node in the documentation.

- A node name: an identifier or name for the node, usually describing the hardware, software or component requiring security services.

- A security triad list: a list of security requirements for the node. Details of security triads are described later.

- Security countermeasure(s): the countermeasures associated with the security triads.

Cables, connectors, connecting accessories, as well as any transmission media used to send information between two components are not represented in the model, and they cannot be represented as infosec-nodes. We consider them only while analyzing transferred information between components. Actually, we only use the end-points at the connected elements. For example, a logical or physical cable connecting a web server and a database server is not an infosec-node, but the servers themselves are, and the two end-points of the connecting cable can be used to implement security controls for information moving in and out of the servers.

Fig. 2 shows an example of an infosec-node, its security requirements and countermeasures. In Fig. 2a the circle represents the component by itself with its internal security requirements (internal triads), and the small black square at the border represents security requirements for information exchanged (end-point triad) with a different node or an element external to the system.

2.2.3. Security triads. A security triad is an element located inside an infosec-node that fully represents a security requirement. A security triad is composed of three items, namely:

- An assurance moment.

- An information state.

- A security service.

Assurance moments add the time dimension to the model. Time is relative to the occurrence of security events. The possible types are protection, detection, and response. These are defined in [22] to represent the time before, during, and after event occurrence, respectively.

Information states represent status of information at different points in time, such as stored

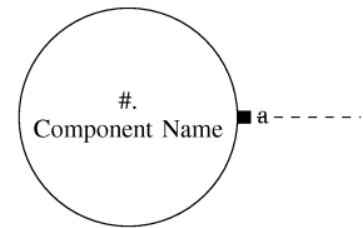

(a) Infosec node.

\begin{tabular}{|c|}
\hline Internal \\
\hline \hline (protection, storage, integrity) $\rightarrow$ countermeasure 1 \\
\hline (protection, storage, confidentiality) $\rightarrow$ countermeasure 2 \\
\hline
\end{tabular}

(b) Infosec-node internal table for $2 \mathrm{a}$.

\begin{tabular}{|c|}
\hline End-point a \\
\hline \hline (protection, transmission, authentication) $\rightarrow$ countermeasure 3 \\
\hline (protection, transmission, confidentiality) $\rightarrow$ countermeasure 4 \\
\hline
\end{tabular}

(c) Infosec-node end-point table for 2a.

Figure 2. Infosec-node details.

information, information being processed, or information being transmitted. We consider the states as defined in [8], and [9]. But we also include additional states considered by [7], namely storage state, processing state, transmission state, creation state, and destruction state.

Security services represent the set of information properties or requirements we want to satisfy when applying security. We consider security services as proposed by several authors [7], [8], [13] to provide a broader scope for the applicability of the model, namely confidentiality, integrity, availability, authentication, non-repudiation, privacy, accountability, and auditability.

As an example, the security triad "(protection, transmission, confidentiality)" represents a requirement to protect (prevent) transmitted information for loss of confidentiality. We use security triads to specify the security requirements of an infosec-node.

Fig. $2 b$ shows requirements of confidentiality and integrity to protect information stored at the node, and countermeasures 1 and 2 are the security mechanisms to enforce them. Fig. 2c shows requirements of confidentiality and authentication to protect information being transmitted in and out of the node, and countermeasures 3 and 4 are the mechanisms to enforce security during transmission.

Infosec-nodes can contain two types of security triads:

- Internal triad: defines a security requirement for information contained, in any state, inside an infosec-node, and independently of its connections with other nodes.

- End-point triad: defines a security requirement for information transferred, i.e. coming in or out of the infosec-node, while exchanged with other infosec-nodes or with elements external to the system.

An infosec-node can have more than one end-point triad, depending on the connections to exchange information with other components. When connections are established with external elements, they typically represent interactions the external 
networks, the Internet, servers, or applications that are not part of the system being assured.

Fig. 3 shows connection examples between the second-level nodes of our example en Fig.1. Servers and storage are physically connected through the networking devices, but we can define a logical connection between them that provides additional security for exchanged information.

\subsection{Methodology Steps}

Defining security requirements is an exhausting and rigorous process that attempts to identify security services and countermeasures, by using security strategies. We define the following steps in the methodology to assure a system using our model.

- $\quad$ Step 1-Gathering security policies and system components.

- $\quad$ Step 2-Constructing the Infosec-tree structure.

- $\quad$ Step 3- Determining triads and countermeasures.

- $\quad$ Step 4- Assuring security controls.

2.3.1. Step 1 - Gathering security policies and system components. This is a preparation step before applying the model. It requires collecting information about the security policies defined for the system and a full inventory of its components, such as hardware, software, connections, services, and so on. We are interested on all the components and connections that handle information in any way, because they are going to be considered when defining the security model.

The typical inputs required to complete this step are:

1. A list with hardware, software, and any physical or logical components belonging to the system, that can be target of an information assurance process.

2. A map describing the connections and interactions between components. For infrastructure systems, it can be a topology map or a diagram, describing real and virtual interactions. On software systems, it can be a diagram or documentation describing interactions between software components, such as UML diagrams or any equivalent document.

3. A list of services provided by the system. This information can help constructing the Infosec-tree structure, and the interactions with external components.

4. A list of services for managing the system. This is a complimentary list describing components and security requirements for the management infrastructure.

5. A document with security policies for information management of the organization.

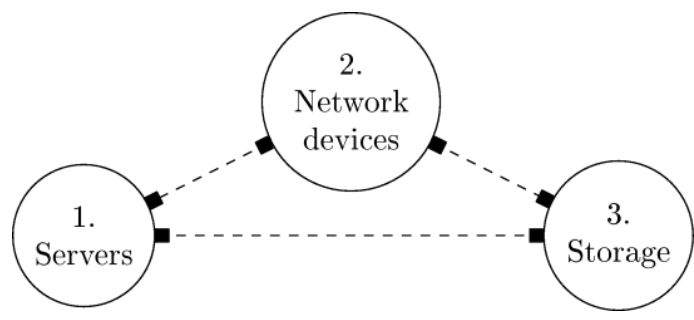

Figure 3. Communicating infosec-nodes.

The documentation collected in this step enables the creation of the hierarchical structure, as described in next step.

2.3.2. Step 2 - Constructing the Infosec-tree structure. We propose two approaches to construct the Infosec-tree, namely (a) a top-down approach, and (b) a bottom-up approach.

The top-down approach starts with a "root" component representing the entire system. We grow down the tree by considering smaller sub-components that handle information. These components are the target to establish the desired security goals. We create new nodes by considering the security requirements of the corresponding components of the system. More detailed nodes allow for more specific security countermeasures to be installed at smaller components.

Nodes of the tree can represent components of several different types, such as physical, logical, real, virtual, hardware, software, or even a mix of them. For example, we can define a node to represent a set of services provided by the system, or a node to represent hardware components and virtual elements all together, but a node can also represent a single small piece of software. The important concept to remember about nodes is that they belong to the tree because they represent system components with security requirements, and they are going to guide the assurance process.

On the other side, the bottom-up-approach starts with lower-level nodes, describing smaller components of the system, and goes up defining nodes for larger integral components that have common security requirements, for all the contained sub-components.

The approaches are not exclusive from each other. We can design portions of the tree using one way or the other, and later put them together at different sections of the tree.

It is also important to mention that creating the tree is an iterative process. We usually start considering the more relevant elements, and later we split them, adding more components, as a result of refining the level of detail while considering the required security for the system.

2.3.3. Step 3 - Determining triads and countermeasures. This step comprises definition of security triads, and the corresponding countermeasures to attend security requirements of the nodes. The step requires iterating over all the nodes, defining the security requirements, the security triads, and finally the security countermeasures. 
To achieve this goal, the Infosec-tree structure facilitates the process of applying security design principles [13], and security patterns [23] in a natural and intuitive way. Multiple lines of defense, layered design, or diversity of mechanisms strategies can be easily applied using the nested structure of components represented by the Infosec-tree.

Upper-level nodes can provide common and general security mechanisms to all their contained components, and lower-level nodes can specify more specific and specialized security controls.

Furthermore, end-point connections are good places to apply strategies such as least privilege, least common mechanism, separation of privilege, or more complex patterns like virtual private network. For internal nodes, we can consider strategies like fail-safe defaults, complete mediation, economy of mechanism, open design, and more.

How and where these principles and patterns are applied to the tree is going to depend on the specific system and security requirements, but the proposed tree structure contributes positively to engage and implement them during the assurance process.

Consider one more time the data center example of Fig. 1, and the following confidentiality policy, information owned or guarded by the organization should be accessed only by authorized people. If there is doubt about authorization, access should be denied. Suppose that we want to protect information for confidentiality while is stored, processed or transmitted. Having a root node to represent the entire data center, we can install a security countermeasure to satisfy this requirement at the root node. This countermeasure is going to protect all the information contained in the corresponding component. But we can also implement additional confidentiality controls for the subcomponents of the data center, i.e. servers, switching device, storage, and recursively apply the same process to the subcomponents of these components.

Now, suppose that we want to apply the same confidentiality policy to a web application running inside a small group of virtual servers, contained in a blade server of the data center. We can use firewall rules at each of the virtual servers to control internal communications between them. But can go one level up, and define a firewall at the network level to control that communication. We can also use both controls, to provide different granularity levels of isolation.

After establishing the security services for the nodes, next step is to create security triads. We have to define the assurance moment (protection, detection, response), the information state (storage, processing, transmission, creation, destruction), and the security service for each triad. To finish this step, we associate an appropriate countermeasure to each triad, according to the security designer decision and to the available security resources. Fig. $2 b$ and Fig. 2c show triad examples with associated countermeasures.

2.3.4. Step 4 - Assuring security controls. This final step is a complimentary process to verify the security of the security countermeasures installed after executing the previous methodology steps. Newly added security components can bring new vulnerabilities for several reasons. They can be complex systems requiring configuration, such as an intruder detection system. They may not be properly configured, or they can have vulnerabilities from factory, that we have to patch before production.

For example, a corporate antivirus platform typically requires some tuning, and periodic maintenance to preserve their integrity and correct functionality. A similar case happens with appliances and security devices that require software, or firmware updates, to fix vulnerabilities, and other functional and security issues.

The process to assure the security countermeasures, when required, starts by adding them to the Infosec-tree. They have to be located in the tree accordingly, like any other component of the system.

After including the security controls in the tree, we have to execute step 3 of methodology for each of them, namely establishing security services, security triads and countermeasures, like any other component of the system.

Next section describes a case study for Infosec-tree model, and some results from applying the methodology to the UCR Academic Cloud.

\section{Study Case: Academic Cloud at UCR}

UCR is the largest and most important public university in Costa Rica. Established in 1940, it has defined goals for education, research and social welfare. UCR academic cloud is an institutional research project started on 2013 to provide the academic and administrative communities at the University with a private cloud computing infrastructure.

Current installed hardware platform has more than 80 half height HP blade servers. Each server contains two eight-core processors, 128 Gbytes RAM, and two 146 Gbytes SAS RAID-1 configured hard disks.

Networking infrastructure connects the cloud to the campus network. It includes two HP 12500 Switches with optional Software Defined Networking (SDN) capabilities. These devices provide fault-tolerant and scalable operation with a throughput of $24.3 \mathrm{Tbps}$.

Cloud storage is powered by an EMC VNX 5700 with a hierarchical disk structure of NL-SAS, SAS, and SSD hard disks, and a raw estimate capacity of 400 TBytes. Storage components provide unified storage for files, blocks and objects, de-duplication, compression and thin provisioning for capacity improvement.

We have chosen this infrastructure to test our methodology because of its complexity and diversity of security requirements. In this section, we describe some real use cases extracted from our security solution for this cloud. 


\subsection{Information Security Policies}

Security policies are the starting point of the assurance process. They are used as the input to apply the methodology. The following is a subset of the security policies at UCR.

1. Confidentiality policy: information owned or guarded by the UCR should be accessed only by authorized people. If there is doubt about authorization, access should be denied.

2. Integrity policy: information stored, transmitted or processed by the UCR systems, and by the effects considered its property cannot be under any circumstances modified or eliminated, without having formal authorization by the authorized personnel.

3. Availability policy: the UCR will provide a continuity plan to reduce the effect of interruptions, so availability of resources and services can be maximized, according to the possibilities of the institution.

4. Authentication policy: information stored, transmitted or processed by the UCR systems, and by the effects considered its property cannot be under any circumstances be accessed without authentication of authorized staff.

\subsection{Case Analysis}

Use cases explained in this section are representative of the assurance process, but they are partial examples. They describe only a small number of the required security services for the real platform. The full process generates extensive documentation that cannot be included in this article.

We start with a high level example describing the root element of the Infosec-tree, and then it comes with more detailed examples.

\subsubsection{Case Analysis for the Top Level Component.}

The first case considers the two upper-levels of the Infosec-tree. Our cloud is composed of a set of servers, networking devices and storage devices, as shown in Fig. 4a.

Our goal is to protect information being transferred in or out of the cloud for confidentiality (policy 1), and also to protect information being transferred inside the cloud for integrity (policy 2).

We can apply security mechanisms at the root node, and also enforce confidentiality at the networking devices. Although, we considered confidentiality and integrity for Servers and Storage, we decided to use the mechanisms at lower-level sub-nodes. The resulting partial Infosec-tree is shown in Fig. 4a.

Now, we can define security triads and countermeasures at the entry point of the cloud. For information coming in and out we have the end-point and the triads shown in Fig. $4 \mathrm{~b}$ and Fig. 4c, respectively. These triads propose a firewall for confidentiality, and an intrusion prevention system

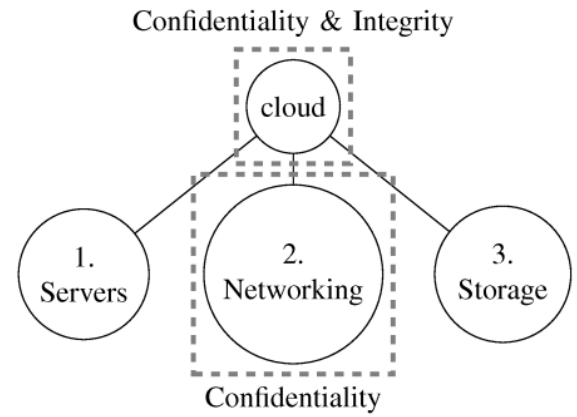

(a) Infosec-tree for the cloud study.

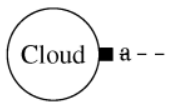

(b) Cloud infosec-

node.

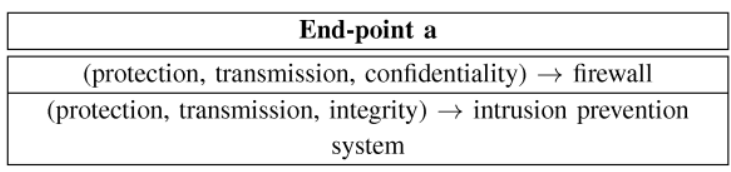

(c) End-point table for Cloud infosec-node.

Figure 4. Case analysis for the cloud component.

(IPS) for integrity. We can install these devices at the entry point of the cloud to enforce the security policies.

From the example we can see that not every end-point has a connecting peer inside the system, namely another component to communicate that belongs to the system. In this case the other end is an external network, mostly the Internet, connected to the cloud data center.

2.3.4. Case Analysis for Storage. This case shows how the whole-parts pattern can be applied into the tree for logical components, as opposed to physical components. The Storage sub-tree shown in Fig. 5a proposes confidentiality, integrity and availability services (policies 1, 2, and 3).

Storage node is composed of two logical subcomponents, namely Storage Area Network (SAN) for local storage, and Network-Attached Storage (NAS) for network storage. They require confidentiality because we want to allow storage access only for blade servers located in the data center, and not for external servers.

About integrity and availability, the hardware capabilities of the storage provide a "pool" abstraction, which allows defining groups of disks, assigned by demand, and they are composed of one or more arrays of physical disks. The hardware also provides RAID technology for arranging the disks. Then, we used these facilities to satisfy the security requirements for integrity and availability.

Fig. 5a shows several types of arrays, such as Performance (Perf. RAID), and Capacity (Cap. RAID), for faster access or larger capacity, respectively. Fig. 5b shows the security triads for Performance RAID node 3.1.1.1. We used the security tools coming with the hardware to configure the countermeasures.

3.2.3. Case Analysis for Production Servers. Last case describes the Servers sub-tree shown in Fig. 6. 


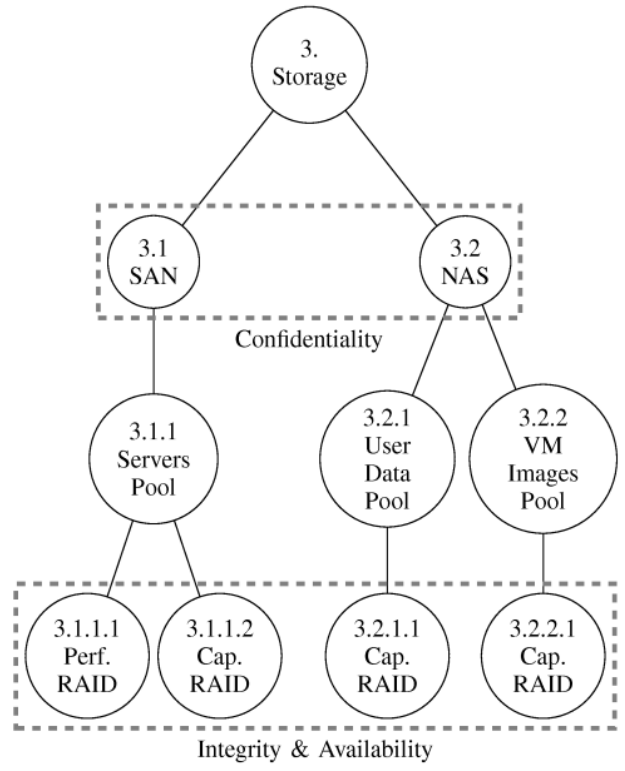

(a) Infosec-tree for Storage showing confidentiality, integrity \& availability services.

\begin{tabular}{|c|c|c|}
\hline \multicolumn{3}{|c|}{ Internal } \\
\hline \multirow{4}{*}{ (protection, storage, integrity) } & & RAID 5 for SAS \\
\hline & $\rightarrow$ & Disks \\
\hline & & RAID 6 for \\
\hline & & NL-SAS Disks \\
\hline \multirow{4}{*}{ (protection, storage, availability) } & & RAID 5 for SAS \\
\hline & $\rightarrow$ & Disks \\
\hline & & RAID 6 for \\
\hline & & NL-SAS Disks \\
\hline
\end{tabular}

(b) Internal triads and countermeasures for infosec-node 3.1.1.1.

Figure 5. Case analysis for storage.

This example considers authentication requirements (policy 4). The Servers node represents the entire set of blade servers, and it is composed of Enclosures.

. Enclosures are grouping nodes. They are real hardware components, with up to 16 blades each. There are several purposes for servers contained in the Enclosures, namely Production, Academy and Management Servers. The servers contain virtual machines, and the virtual machines provide network services to the users.

Enclosures and Blade Servers can be managed remotely, and we want to apply the authentication policy. We decided to implement two authentication services, one for management the cloud (core authentication), and the other for final users (institutional authentication).

In this example, the authentication servers are also included into the Infosec-tree, because of their inherent complexity, as described in step 4 of the methodology, so they can be target of the assurance process too.

Next section describes different approaches for security information and assurance, which relate to our model and methodology.

\section{Related Work}

Existing information security concepts, and other security approaches can help to detail the context of the work presented in this paper. We

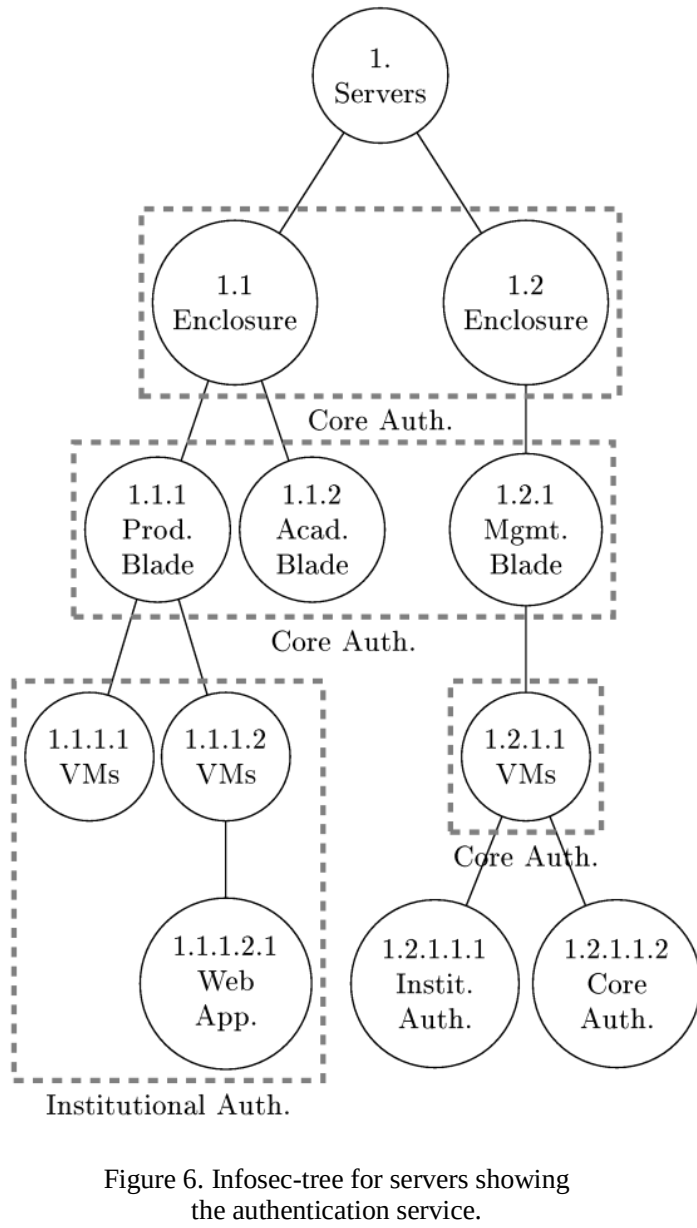

provide an overview of basic security definitions, relevant security models, and industry standards. A comparison of some of these models with the Infosec-tree model is presented in Section 4.3.

\subsection{Information Security Models and Standards}

Information security is about protecting information for confidentiality, integrity, availability, authenticity, non-repudiation and other additional security goals [7], [13]. Information security policies provide a framework to specify decisions for accessing and protecting information in a system. Moreover, information security models are tools to enforce security policies using formal or informal methods. Security standards capture generalized practices to help reducing the number of successful attacks directed towards information in a system.

ISO/IEC 27000 standard series [14] contain high level guides, with best practices and recommendations for information security management, and they focus on risk and security management for an organization.

Control Objectives for Information and Related Technology (COBIT) [15] incorporates enterprise governance, management techniques, and provides guides for information security and assurance, based on five principles, and seven enablers to achieve the business objectives. 
The National Institute of Standards and Technology (NIST) [16], [17] also provides a large number of publications with guidelines, best practices and standards related to computer security.

Some models focus on information security, such as McCumber security model [9]. This model defines a framework for information assurance. It considers security goals (confidentiality, integrity, and availability), information states (processing, storage, and transmission) and security countermeasures (technology, policy and practices, human factors), using a three-dimensional cube to represent potential security goals to be satisfied.

Maconachy [8] proposes a modified version of McCumber model by renaming security goals into security services, adding authentication and non-repudiation to the security services, and a new time dimension for the assurance process.

Table 1: Comparison of standards and security models, in the context of information security.

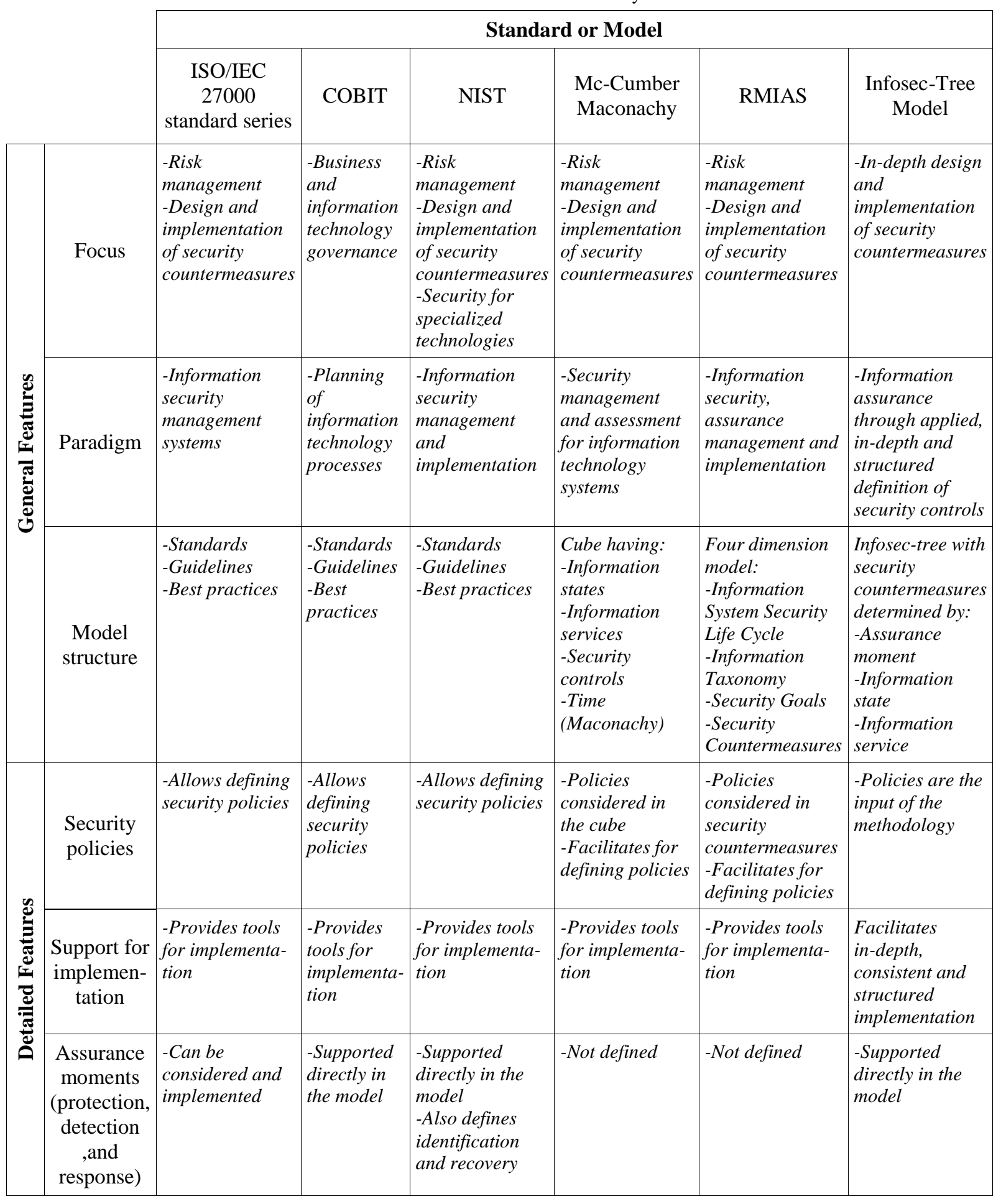


Reference Model for Information Assurance \& Security (RMIAS) [7] is a related model, which considers information security life cycle, information taxonomy, security goals, and security countermeasures. It also includes details for location, sensitivity, and format, such as paper, electronic, or verbal information.

\subsection{Security in Cloud Computing}

Regarding to the cloud use cases, we looked for security of large and complex infrastructures. Cloud platforms suffer most challenges and issues found in traditional information technology infrastructures. But they also come with new risks, threats and challenges [18] that may slow down the acceptance as an emerging technology [19].

Academia researchers describe relevant security challenges for clouds [6], [7], [10], [12], [20], [21]. Authors on [20] mention information security as a research issue, while other researchers [10], [11] study the problem using a more theoretical approach.

Important security challenges have been introduced when migrating traditional data centers towards cloud computing, because the conversion process adds new complexities and diminishes the effectiveness of conventional protection mechanisms. For example challenges related to virtualization [3] and authentication technologies [18], [21].

\subsection{Comparison of security standards and models}

We compared some security models and standards with our Infosec-tree model. Comparison results are shown in Table 1 .

We defined two groups of features as the comparison criteria, namely general features (focus, paradigm and model structure), and detailed features (security policies, support for implementation, and assurance moments). The features describe some important difference between the models. The comparison goal is to show differences between models, but we are not looking for a winner standard.

Table 1 shows important benefits of the Infosec-tree Model when compared with existing security models and standards. The assurance process is designed to support practical implementations. The assurance steps are structured, in-depth and consistent, given a pre-defined set of security policies. The methodology can be used to satisfy security requirements for protecting, detecting, or responding to security events.

\section{Conclusions}

Information security and assurance are complex fields. Assuring large modern technological systems is also complex. We propose a model and a methodology to deal with complexity using a divide-and-conquer idea, and we used a practical tree structure as the main tool.

We applied this idea to our cloud at the university, and the problem became clear and manageable. The methodology provided a convenient tool to deal with the inherent complexity, for information security and the assurance process. The cloud will grow and we expect to keep the assurance process still manageable.

We have also applied the methodology to design security of software applications, mostly for research projects with students, and the process is analogous. Hardware devices, virtual appliances, other components, and their connections become software modules, objects, functions, and interactions between them.

So far, for assuring information, our methodology shows an intuitive and consistent process, independently of the nature of the components managing the information.

Furthermore, the methodology by itself is a documentation system for security requirements and countermeasures, and the documentation structure is also modular enough that it can be updated very easily over time.

\section{References}

[1] C. Gong, J. Liu, Q. Zhang, H. Chen, and Z. Gong, "The characteristics of cloud computing", in Parallel Processing Workshops (ICPPW), 2010 39th International Conference on. IEEE, 2010, pp. 275-279.

[2] P. Mell and T. Grance, "The NIST definition of cloud computing”, National Institute of Standards and Technology, vol. 53, no. 6, p. 50, 2009.

[3] T. Erl, R. Puttini, and Z. Mahmood, "Cloud Computing: Concepts, Technology \& Architecture", 1st ed. Upper Saddle River, NJ, USA: Prentice Hall Press, 2013.

[4] S. Subashini and V. Kavitha, "A survey on security issues in service delivery models of cloud computing", Journal of Network and Computer Applications, vol. 34, no. 1, pp. 1-11, 2011, http://www.sciencedirect.com/science/article/pii/S10 84804510001281 (Access Date: 15 Jul, 2014).

[5] T. Dillon, C. Wu, and E. Chang, "Cloud computing: Issues and challenges", in Advanced Information Networking and Applications (AINA), 2010 24th IEEE International Conference on. IEEE, April 2010, pp. 27-33.

[6] K. Bhamidipati and S. Karanth Shyam, "The need, use and efficiency of trustworthy security model in cloud computing for information assurance", in Cloud Computing Technologies, Applications and Management (ICCCTAM), 2012 International Conference on. IEEE, 2012, pp. 69-73.

[7] Y. Cherdantseva and J. Hilton, "A reference model of information assurance \& security", in Availability, Reliability and Security (ARES), 2013 Eighth International Conference on. IEEE, 2013, pp. 546-555. 
[8] W. V. Maconachy, C. D. Schou, D. Ragsdale, and D. Welch, "A model for information assurance: An integrated approach", in Proceedings of the 2001 IEEE Workshop on Information Assurance and Security, vol. 310. New York, USA, 2001.

[9] J. McCumber, "Information systems security: A comprehensive model", in Proceedings of the 14th National Computer Security Conference, 1991.

[10] S. Ristov, M. Gusev, and M. Kostoska, “A new methodology for security evaluation in cloud computing", in MIPRO, 2012 Proceedings of the 35th International Convention. IEEE, 2012, pp. 1484-1489.

[11] L. B. A. Rabai, M. Jouini, A. B. Aissa, and A. Mili, "A cybersecurity model in cloud computing environments", Journal of King Saud University Computer and Information Sciences, vol. 25, no. 1, pp. 63 - 75, 2013, http://www.sciencedirect.com/ science/article/pii/ S131915781200033X (Access Date: 2 Aug, 2014).

[12] K. S.O., I. F., and O. Awodele, "Cloud computing security issues and challenges", International Journal of Computer Networks, 2011.

[13] M. Bishop, "Computer security: art and science”, Addison-Wesley, 2002.

[14] ISO, "ISO 27005:2008 Information technology - Security techniques - Information security risk management”, ISO, 2008.

[15] Information Systems. Audit and Control Association, "COBIT 5 for Information Security Std.”, 2013.

[16] National Institute of Standards and Technology, "FIPS 46-3: Data encryption standard”, Federal Information Processing Standards, National Bureau of Standards, US Department of Commerce, 1999.

[17] National Institute of Standards and Technology, "FIPS 140-2: Security requirements for cryptographic modules", Federal Information Processing Standards, National Bureau of Standards, US Department of Commerce, 2001.
[18] S. Mansfield-Devine, "Danger in the clouds”, Network Security, vol. 2008, no. 12, pp. 9 11, 2008. [Online]. Available: http://www.sciencedirect.com/science/article/pii/S13 53485808701405 (Access Date: 23 Jun, 2014).

[19] F. Gens. (2009, December) "New idc it cloud services survey: Top benefits and challenges". IDC eXchange. [Online]. Available: http://blogs.idc.com/ie/?p=730 (Access Date: 02 Aug, 2014)

[20] M. Ahmed, A. S. M. R. Chowdhury, M. Ahmed, and M. M. H. Rafee, "An advanced survey on cloud computing and state-of-the-art research issues", IJCSI International Journal of Computer Science Issues, vol. 9, no. 1, January 2012.

[21] D. Zissis and D. Lekkas, "Addressing cloud computing security issues", Future Generation Computer Systems, vol. 28, no. 3, pp. 583 - 592, 2012, http://www.sciencedirect.com/science/article/ pii/S0167739X10002554 (Access Date: 23 Jun, 2014).

[22] R. Bejtlich, "The Tao of Network Security Monitoring: Beyond Intrusion Detection”, Pearson Education, 2004.

[23] E. Fernandez-Buglioni, "Security Patterns in Practice: Designing Secure Architectures Using Software Patterns”, 1st ed. Wiley Publishing, 2013.

[24] R. Villalón, B.Solano, and G.Marín, “An Applied Methodology for Information Security and Assurance”, International Conference for Internet Technology and Secured Transactions, 2014.

[25] V. Arora, "Comparing different information security standards: COBIT v s. ISO 27001, 2010”, [Online]. Available: https://qatar.cmu.edu/media/ assets/CPUCIS2010-1.pdf (Access Date: 27 Dec, 2014)

\section{Acknowledgements}

We would like to acknowledge the entire university personnel participant in any form in the CITIC-ECCI Research Project 834-B3-145 "Creación de la Nube Académica Computacional de la UCR” for the continuous support and hard work. 